\title{
Editorials
}

\section{Integrating primary mental health care and mental health promotion}

The mental health theme in this issue of the BJGP comes at a time of growing awareness of the need to integrate mental health services within the UK NHS. The 2013 Annual Report of the Chief Medical Officer (CMO) ${ }^{1}$ reminds us that mental illness is the largest single cause of disability, representing $28 \%$ of the national disease burden in the UK. It is the leading cause of sickness absence in the UK, accounting for 70 million sick days in 2007. Mental illness costs the UK economy $€ 70-€ 100$ billion per year; $4.5 \%$ of gross domestic product. People with mental illness die on average 15-20 years earlier than those without mental illness, often from avoidable causes. About 75\% of people with mental illness receive no treatment at all. The CMO Report describes the need for integrated mental health care throughout the life course: children and young people, adults, and older adults.

Articles in this issue of the BJGP remind us that general practice is involved at every life stage. They come from six European countries and focus on adolescence, older people, healthcare colleagues, and end-oflife care. Haugen and colleagues present a study from Norway and Denmark that found a useful way to screen for depression in adolescents. Their study describes three questions that you may want to ask those who may be depressed. ${ }^{2}$ Hughes and colleagues present a study of patients in Yorkshire (England) aged $16-40$ years who were on antidepressants. Their sample suggests that $7 \%$ may have unrecognised bipolar disorder (for whom mood stabilisers may be helpful) and they propose using the Mood Disorder Questionnaire for those who do not respond well to antidepressants. ${ }^{3}$ Malpass and colleagues used cognitive interviewing with 18 participants to discover that the PHQ-9 questionnaire did not always map onto their personal experiences of depression. ${ }^{4}$ The research team reminds GPs to use clinical judgement when assessing the severity of depression and not merely rely on screening tools. In their study of the impact of structured support in helping people to discontinue benzodiazepines, Vicens and colleagues in Spain found that two-thirds of those who had stopped benzodiazepines at 12 months remained off them at 36 months. $^{5}$ Nic an Fhaili and colleagues explored the role of GPs in Ireland in supporting the

\section{"It will require team-working, strategic partnerships and 'local health communities' for positive mental health promotion for all citizens, as well as care for those who are mentally unwell...}

families of those who had taken their own lives. They remind us that a minimum of six people are affected by every suicide. This study showed that GPs were particularly helpful when they acknowledged the loss and lived life of the deceased, helped to reduce stigma, and directly supported families during the grief journey. ${ }^{6}$ Finally, GPs in all European countries may take note of the study by Verweij and colleagues from the Netherlands that shows that mindfulness training significantly reduces emotional exhaustion in GPs who are suffering from burnout.?

Collectively, these studies affirm the central role of primary care in addressing the mental health challenge at all stages of life. This central role was noted over a decade ago by the much-missed Helen Lester. ${ }^{8}$ In her BJGP article aptly titled 'Integrated primary mental health care: threat or opportunity in the new NHS?' she and her co-authors explained why integration needs to happen at primary and community care level. They wrote:

'[Primary care] occupies an important space at the interface of users, families, communities, and professional worlds and is able to address mental, physical, and social aspects of care. Primary care is also a low-stigma setting, able to offer rapid access for both routine and crisis care, a longitudinal approach to care where patients are never discharged, and perhaps above all, interpersonal continuity of care ...

... Primary care is perhaps better placed to fulfil each of these elements of continuity than secondary care specialist services. Primary care also appears to be generally preferred as a setting by both mental health service users and carers...

This new localism is now NHS policy. In the 2014 Five Year Forward View ${ }^{9}$ the NHS Chief Executive (CE) stated:
'The future will see far more care delivered locally but with some services in specialist centres, organised to support people with multiple health conditions, not just single diseases...

It will require team-working, strategic partnerships and 'local health communities' for positive mental health promotion for all citizens, as well as care for those who are mentally unwell:

- '...newpartnershipswith local communities, local authorities and employers.'

- 'Patients will gain far greater control of their own care."

-... the NHS will become a better partner with voluntary organisations and local communities. ${ }^{\prime 9}$

It will require systematic working across disciplinary and organisational boundaries:

- '... break down the barriers in how care is provided ... between family doctors and hospitals, between physical and mental health, between health and social care."

Achieving primary care leadership of integrated primary mental health care and health promotion is easier said than done. Primary care practitioners may be best placed to orchestrate it, but they are not trained to develop multiple-way partnerships and communities for health. And in any case they are already overwhelmed by other demands.

Even if GPs and other primary care practitioners were to lead integration of health and care, the infrastructure to support it is weak and issues as basic as evaluation have barely been thought of. Public service cuts make realising a collaborative approach across the physical and social dimensions of health more difficult than in previous times, and unprecedented cuts to social care budgets strip the sector's scope for all but its most 


\section{“... integrated primary mental health care and mental health promotion should remain the goal. Despite contemporary difficulties we have to ask: how can collaboration between primary and social care for good mental health best be modelled?"}

pressing statutory obligations. At the same time reducing welfare budgets is creating greater mental ill-health. In this context an agenda of collaboration, prevention, and wellbeing seems optimistic.

Nevertheless, integrated primary mental health care and mental health promotion should remain the goal. Despite contemporary difficulties, we have to ask: how can collaboration between primary and social care for good mental health best be modelled?

London Journal of Primary Care (LJPC) is the journal of the three London Faculties of the Royal College of General Practitioners (RCGP). As editors of LJPC we share the vision of Lester, the NHS CMO, and the NHS CE for sustainably commissioned, integrated primary care-based mental health services, underpinned by new ways of learning and working together. We recognise that it is difficult, but not impossible. To understand how to achieve it, in 2015 LJPC convened an expert panel, in partnership with RCGP and the Educational Trust for Health Improvement through Cognitive Strategies (the ETHICS Foundation). The panel produced three documents to assist thinking about practical ways forward. These can be downloaded free from the internet (http://ethicsfoundation. org/2015/09/04/mental-health-promotionsaves-lives/).

One reality we have to face is that the science of integrated working is a different science from that of the laboratory. We have to use multimethod research to reveal the dynamic interactivity and trusted relationships that lie at the heart of integrated care. Much progress has been made in understanding the complex and adaptive nature of the world outside of laboratories; through languages of integration, complexity, whole systems, organisational learning, qualitative research, comparative case studies, and so on. They reveal how working deliberatively with communities and networks can be understood.

We plan to publish an account of 'Connected Communities', a 5-year study in seven sites that reveals the wellbeing, citizenship, capacity, and economic dividends that community capital can generate. This includes work on parenting, partnership between schools, and clusters of general practices, how music and the arts can heal people and build communities, as well as the social and occupational impacts on mental health at all stages of life lfor example parents, schools, workplace, retirement, the third age, and end of life). Primary care does not have to do all of this, but it does need to orchestrate it. And general practice really does need to step up to the challenge.

\section{Paul Thomas,}

GP. Cuckoo Lane Surgery, West London; Editor-in-Chief, London Journal of Primary Care.

\section{David Morris,}

Professor of Mental Health, Inclusion and Community, University of Central Lancashire; Editor London Journal of Primary Care.

\section{Provenance}

Freely submitted; externally peer reviewed.

DOI: 10.3399/bjgp16X683533

\section{ADDRESS FOR CORRESPONDENCE}

Paul Thomas

Cuckoo Lane Surgery, 1st Floor Hanwell Health Centre, 20 Church Road, London W7 1DR, UK.

\section{E-mail: paul.thomas7anhs.net}

\section{REFERENCES}

Department of Health. Annual Report of the Chief Medical Officer 2013: Public Mental Health Priorities: Investing in the Evidence. DH, 2013. https://www.gov.uk/government/ publications/chief-medical-officer-cmoannual-report-public-mental-health laccessed 31 Dec 2015)

2. Haugen W, Rikard Haavet O, Kaur Sirpal M, Sparle Christensen K. Identifying depression among adolescents using three key questions: a validation study in primary care. $\mathrm{Br} J \mathrm{Gen}$ Pract 2016; DOI: 10.3399/bjgp16X683461.

3. Hughes T, Cardno A, West R, et al. Unrecognised bipolar disorder among UK primary care patients prescribed antidepressants: an observational study. $\mathrm{Br} \mathrm{J}$ Gen Pract 2016; DOI: 10.3399/bjgp16X683437.

4. Malpass A, Dowrick C, Gilbody S, et al Usefulness of $\mathrm{PHQ}-9$ in primary care to determine meaningful symptoms of low mood: a qualitative study. Br J Gen Pract 2016; DOI: 10.3399/bjgp16X683473.

5. Vicens C, Sempere E, Bejarano F, et al. Efficacy of two interventions on the discontinuation of benzodiazepines in long-term users: 36-month follow-up of a cluster randomised trial in primary care. Br J Gen Pract 2016; DOI: 10.3399/bjgp16X683485.

6. Nic an Fhailí M, Flynn F, Dowling S. Experiences of suicide bereavement: a qualitative study exploring the role of the $\mathrm{GP} . \mathrm{Br}$ J Gen Pract 2016; DOI: 10.3399/bjgp16X683413.

7. Verweij H, Waumans RC, Smeijers D, et al. Mindfulness-based stress reduction for GPs: results of a controlled mixed methods pilot study in Dutch primary care. Br J Gen Pract 2016; DOI: 10.3399/bjgp16X683497.

8. Lester H, Glasby J, Tylee A. Integrated primary mental health care: threat or opportunity in the new NHS? Br J Gen Pract 2004; 54: 285-291.

9. NHS England, Public Health England, Health Education England, Monitor, Care Quality Commission, NHS Trust Development Authority. Five Year Forward View. http:// wuw.england.nhs.uk/wp-content/ uploads/2014/10/5yfv-web.pdf laccessed 12 Jan 2016). 